\title{
El uso de marcadores discursivos en la producción lingüística de un paciente con afasia motora aferente
}

\author{
Use of discourse markers in linguistic production \\ of one speaker with afferent motor aphasia
}

Josaphat Guillén E.

Doctor en Lingüística Benemérita Universidad Autónoma de Puebla
Autor correspondiente: Josaphat Guillén Escamilla Puebla - México Correo-e: jguillen@colmex.mx

Recibido: 19/07/2017 Aceptado: 20/09/2017

\section{RESUMEN}

En este trabajo se analiza el uso y las funciones de los marcadores discursivos en la producción de un paciente con afasia motora aferente. Se analiza un corpus proveniente de una entrevista semiestructurada realizada durante la terapia neuropsicológica. El objetivo es analizar cualitativamente el uso de los marcadores discursivos y las funciones que desempeñan a lo largo de las intervenciones del paciente. Se busca mostrar que los marcadores discursivos se conservan en este tipo de afasia porque funcionan como estrategias compensatorias que facilitan la comunicación del paciente y responden a los déficits particulares de la afasia motora aferente. El análisis de los datos muestra que el paciente emplea consistentemente cuatro tipos de marcadores discursivos: (i) conectores, (ii) reformuladores, (iii) formuladores y (iv) modalizadores, a pesar del déficit lingüístico que padece. Esto sugiere que los marcadores desempeñan funciones específicas que contribuyen a subsanar los problemas a los que se enfrenta el paciente durante la construcción de su intervención. Este hecho resulta consistente con los resultados previos obtenidos en otras variedades de afasia. Finalmente, esto contribuye a la idea de que en la afasia el nivel pragmático-discursivo sufre una desintegración menos drástica si se le compara con el nivel fonético-fonológico o el sintáctico.

Palabras clave: marcadores discursivos, afasia motora aferente, estrategias compensatorias.

\begin{abstract}
This paper analyzes use and functions of discourse markers in linguistic production of one speaker with afferent motor aphasia. A semi-structured interview carried out during neuropsychological therapy was analyzed. The aim of this study is to qualitatively analyze the use of discourse markers and the functions they perform in patient interventions. It attempts to show discourse markers are conserved in this type of aphasia because they perform as compensatory strategies which make patient communication easier and respond to the particular deficits which characterize this aphasia. Data analysis shows that the patient consistently uses four kinds of markers: (i) connectors, (ii) reformulators, (iii) formulators, and (iv) modalizers, despite pathology. This suggests that discourse markers perform specific functions which help to resolve the problems which the patient faces during construction of his intervention. This fact is consistent with the results obtained in previous studies with other aphasia varieties. Finally, this contributes to the notion that, in aphasia, pragmatic and discursive levels suffer a less drastic disintegration than that of the phonetic-phonological level or the syntactic level.
\end{abstract}

Keywords: discourse markers, afferent motor aphasia, compensatory strategies. 


\section{Introducción}

En los últimos años, los estudios lingüísticos centrados en el análisis de los déficits del lenguaje en la afasia han ido en aumento; más aún, el marco teórico de la lingüística ha sido incorporado, aunque de forma paulatina, al de la neuropsicología con el fin de realizar estudios interdisciplinares que puedan dar cuenta, de manera más exhaustiva, de los mecanismos neuropsicológicos que se encuentran dañados en esta patología para, posteriormente, establecer sus correlatos lingüísticos. En este sentido, los estudios tradicionales se han centrado en los niveles más básicos del lenguaje -desde el fonético hasta el semántico-, pero en fechas recientes han girado su atención hacia los niveles pragmático y discursivo, principalmente porque han demostrado ser de utilidad para repensar las clasificaciones tradicionales de las afasias (Pietrosemoli, 1994) y porque pueden plantearse instrumentos de evaluación para el componente pragmático-discursivo (Gallardo-Paúls, 2009), tal y como existen para los demás niveles de análisis lingüístico (Basso, 2003). Justamente, este trabajo aborda el análisis de uno de estos fenómenos, en específico, los marcadores discursivos (MD).

\section{Los marcadores discursivos}

Los MD son elementos lingüísticos que utilizan los hablantes para cumplir diversas funciones, entre ellas, señalar la forma en la que la unidad anterior de habla se relaciona con el estado actual del discurso (Schiffrin, 1987). Pueden ser palabras o frases que dividen, ordenan, estructuran y muestran la relación que guardan entre sí las partes del discurso, ya sean ideas, acciones, turnos, etcétera. Portolés (1998) los define como: "unidades lingüísticas invariables, [que] no ejercen una función sintáctica en el marco de la predicación oracional y poseen un cometido coincidente en el discurso: el de guiar, de acuerdo con sus distintas propiedades morfosintácticas, semánticas y pragmáticas, las inferencias que se realizan en la comunicación" (p. 27). Así, esta dependencia secuencial indica que trabajan en un nivel discursivo más que en el de la palabra o la oración.

Ahora bien, también pueden desempeñar otras funciones, puesto que, a través de este tipo de operadores pragmáticos, "'el hablante justifica la conclusión, su opinión', 'cambia el tema de conversación o lo redirige', 'el hablante llama la atención sobre algo o realza algo', 'expresa un falso acuerdo', 'controla el contacto o interpela', 'señala que su turno continúa', 'marca el inicio del diálogo o lo cierra', etc., y todo ello ante un oyente o lector" (Briz, Pons y Portolés, 2008). Todas estas funciones sugieren que los MD contribuyen tanto a la coherencia y cohesión, como al manejo de la conversación, por lo que suelen dividirse en tres grandes tipos: (i) conectores, (ii) modalizadores y (iii) controladores de contacto.

Esta investigación se centra en los dos primeros tipos, pues se parte del supuesto que el paciente utiliza los conectores para: señalar el tipo de relación argumentativa entre dos partes del discurso, redirigir la conversación, corregir o parafrasear lo dicho, ganar tiempo para formular su turno, marcar el inicio del turno, señalar que su turno continúa y, con todo esto, asegurar el orden y la organización discursiva. 
Por su parte, a través de la modalización, es capaz de justificar su opinión y expresar un falso acuerdo. En contraste, el empleo de controladores de contacto será muy poco probable, pues la toma y cesión de turno están relativamente bien delimitadas por las pausas $y$, sobre todo, por los silencios prolongados del hablante.

Así pues, a continuación se describirán brevemente cuatro tipos de marcadores discursivos: (i) conectores, (ii) reformuladores, (iii) formuladores y (iv) modalizadores. En especial, se destacarán las funciones que desempeñan en el discurso de normohablantes para, posteriormente, determinar si el paciente los utiliza de forma convencional o no.

\section{Conectores}

De acuerdo con la distinción propuesta por Pons (2001, p. 230-231), dentro de la función de conexión se distinguen dos subfunciones: (i) una argumentativa, como el resultado conjunto de las propiedades argumentativas e inferenciales de un conector (Anscombre y Ducrot, 1994) y otra (ii) función metadiscursiva (Briz, 1993), que trata con la construcción del discurso -reformulación y formulación-.

Los conectores argumentativos funcionan principalmente para que los hablantes articulen sus estrategias comunicativas y señalen el tipo de relación que establecen los dos miembros del discurso que unen. Son guías inferenciales que trabajan en un nivel argumentativo de conexión, auxiliados en muchos casos por el significado convencional del marcador mismo; por ejemplo, el de oposición de pero, el de causa de porque o pues, el consecutivo de por eso o entonces, etcétera. Lo que hay que destacar es que estos marcadores conectan argumentos más que oraciones.

(1) A: Juan es joven, pero tiene experiencia.

(2) B: Juan tiene experiencia, pero es joven.

En (1) y (2) lo que varía es la orientación argumentativa de los enunciados, esto es, pero une dos enunciados que guían a conclusiones opuestas: si el que antecede al marcador se dirige hacia una conclusión negativa, el segundo lleva a una conclusión positiva, como en (1), y viceversa, como en (2). Así, este tipo de marcadores, por el simple hecho de aparecer, obliga a releer los argumentos y guía el proceso inferencial que debe realizar el oyente.

\section{Reformuladores}

Durante la conversación, los hablantes se enfrentan a diversos tipos de problemas, los cuales deben ser reparados para que el intercambio comunicativo pueda seguir. Una de estas dificultades son los errores que se cometen al decir algo impreciso, equivocado o inadecuado. Cuando esto ocurre, los hablantes pueden emplear diversos dispositivos lingüísticos para realizar la corrección, entre ellos, los reformuladores. Estos marcadores están relacionados con la corrección de movimientos discursivos, con "el proceso de distanciamiento que, en ocasiones, mantiene el hablante con respecto a lo dicho, y que implica que lo que éste dice después del reformulador es el segmento más importante para la continuación del discurso" (Pons, 1998, p. 205). 
(3) A: Juan avisó que estaba saliendo de la oficina, o sea, apenas está apagando la computadora.

(4) B: Siempre he pensado que casarse es de locos, bueno, de valientes.

En (3), el hablante A parafrasea lo dicho en un primer momento, "estar saliendo de la oficina", y lo corrige por lo que realmente estaba haciendo Juan, "estar apagando la computadora". Esta corrección es introducida por el reformulador o sea. En el caso de (4), el hablante B se distancia de su propia opinión sobre el matrimonio, "una cosa de locos", y la matiza en el enunciado introducido por el reformulador bueno, al considerarlo como algo "de valientes". Así pues, la función de estos marcadores está directamente relacionada con la corrección, explicación, paráfrasis o aclaración de ideas o argumentos expresados en unidades discursivas previas (Pons, 2001, 2003; Briz y Pons, 2010). Los ejemplos más representativos de estos marcadores son: esto es, es decir, o sea, en otras palabras, bueno, etc.

\section{Formuladores}

Esta función está relacionada con la forma en la que se construye el mensaje y los problemas relacionados con la planeación y construcción del turno conversacional. Estos problemas son de diversa índole: falta de atención, sobrecarga cognitiva, déficits de memoria, etcétera. Entonces, este tipo de conectores pone de manifiesto los titubeos, las dudas y los reparos que sufren los hablantes durante la construcción del mensaje. Esta clase de marcador le permite al hablante asegurar su participación, ya que ayuda a retrasar la planeación y construcción de la intervención, indicando que aún no ha concluido o que está por hacerlo. De lo anterior se desprende que sean considerados como pausas oralizadas cuya función principal es la de mantener el turno conversacional.

(5) A: ¿Cómo llego a la avenida principal?

B: puees, lo primero que tiene que hacer es girar a la derecha y seguir derecho tres calles más.

(6) C: Es sencillo, comienzas cortando el pedazo de madera, luego lo vas modelan- le das forma con el esmeril basándote en el, en el, bueno, siguiendo en el modelo.

En (5), el hablante B retarda su intervención apoyado por el formulador pues, lo que le sirve para ganar tiempo y pensar la respuesta que va a dar. Esto se ve reforzado por el alargamiento vocálico y la pausa posterior al marcador. En cuanto a (4), el hablante tiene problemas para construir su intervención y duda de lo que iba a decir, así que utiliza el marcador para solventar estas dificultades; además, aparte del marcador, hay otros elementos que señalan estos contratiempos: la repetición de "en el" y las pausas antes y después del marcador.

Gracias a lo anterior, a estos marcadores "se les ha asignado un papel retardatorio; fórmulas, así pues, que permiten ganar tiempo para pensar $y$ 'planificar' lo que se va a decir a continuación; pausas para pensar, elementos que rellenan espacios vacíos cuando el hablante inicia un discurso, cuando no encuentra el modo de continuar" (Briz, 1993, p. 45). Entre los ejemplos más representativos están: 
bueno, pues, eh, este, esto, etcétera.

\section{Modalizadores}

La modalidad está relacionada con un énfasis, una atenuación, un acuerdo o desacuerdo, por lo que está íntimamente ligada a la actitud del hablante con respecto al enunciado y la forma en que se enfrenta al mensaje (Briz, 2008; Pons, 1998). Así, la modalidad puede ser entendida como la actitud subjetiva del hablante con respecto al contenido de la enunciación (Briz, 2008) y puede indicar, entre otras cosas, (i) el grado de certeza o duda que tiene el hablante acerca de lo que dice, (ii) la opinión del hablante y (iii) un falso acuerdo. Por ejemplo:

(7) A: los gatos son ariscos, por eso no me gustan.

B: bueno, pero no todos, habrá algunos que no.

(8) C: como siempre, llegando tarde.

D: es que perdí el autobús.

En (7), el hablante B concede parcialmente la razón a $A$, esto es, bueno indica un falso acuerdo, función que se ve reforzada con la aparición de marcador pero. En tanto que en (8), D no sólo explica el motivo por el que llegó tarde, sino que fundamentalmente justifica su falta. Algunos ejemplos de estos marcadores son: a decir verdad, a lo mejor, bueno, mira, hasta, de plano, en verdad, de hecho, etcétera.

Así pues, se han presentado las unidades de análisis lingüístico que se van a estudiar en la producción de un paciente con afasia motora aferente. Como ya se dijo, el objetivo es comprobar si el uso de este tipo de marcadores se mantiene y si su empleo es productivo en este tipo de afasia. En otras palabras, buscamos determinar si estos MD sirven como estrategias compensatorias comunicativas que ayudan, en la medida de lo posible, a subsanar y a lidiar con las carencias lingüísticas que padece el paciente.

\section{Afasia motora aferente ${ }^{1}$}

En ciertas tradiciones epistemológicas, como la de Geschwind (1976) y Goodglass y Geschwind (1976), suele definirse un solo síndrome afásico motor que se caracteriza por una alteración general en la capacidad para articular fonemas, sílabas y palabras, aun cuando se puedan preservar otros tipos de movimiento de la cavidad orofacial (Quintanar, Lázaro García y Solovieva, 2006). En contraste, la tradición neuropsicológica soviética establece una diferencia entre dos tipos de afasia motora, fundamentalmente porque esta patología puede ocurrir a partir del daño en dos áreas cerebrales distintas, alterando factores neurológicos

\footnotetext{
${ }^{1}$ Otros nombres para este tipo de síndrome son: afasia central y afasia de conducción aferente. En este sentido, cabe aclarar que, más allá de las diferencias taxonómicas: "the distinction between conduction aphasia and afferent motor aphasia remains a matter largely open to discussion" (Valdois, Joanette, Nespoulous y Poncet, 1988, p. 60). Por ejemplo, Ardila (2005) sostiene que existe un paralelo entre ambas afasias, dado que comparten las características clínicas y el tipo de déficit lingüístico; así pues, la afasia motora aferente correspondería a una afasia de conducción, donde "el lenguaje espontáneo puede fluctuar y en ocasiones es fluido, y en otras ocasiones no fluido, parafásico y difícil en su producción [...] Estrictamente hablando, se podría considerar como una afasia fluida o como una afasia no fluida, aunque usualmente se interpreta como una afasia fluida (Ardila, 2005: 100). No obstante, el trabajo de Valdois et al. (1988, p. 8788) demostró que ambas afasias presentan una diferencia fundamental: la aferente viene acompañada de una severa apraxia bucolingual, mientras que la de conducción no, por lo que muestran dos patrones distintos en el tipo de error. Así, concluyen que la afasia de conducción no es un síndrome homogéneo, por lo que la afasia motora aferente puede ser considerada como una forma particular de afasia de conducción, una no fluida. Con todo, el debate aún sigue abierto.
} 
subyacentes también distintos (Luria, 1964). En específico, un daño en la parte inferior de la región postcentral del hemisferio izquierdo origina una afasia motora aferente -comprometiendo la integración cinestésica-, mientras que un daño en regiones frontales posteriores del mismo hemisferio provoca una afasia motora eferente -alterando la melodía cinética ${ }^{2}$-.

En consecuencia, para Luria (1964, 1977), la nomenclatura de afasia motora es muy vaga y no captura, a detalle, las "sutiles" diferencias entre: (i) encontrar los movimientos articulatorios necesarios para la producción de lenguaje expresivo y (ii) la organización serial de dichos movimientos articulatorios (Quintanar et al., 2006).

\section{Cuadro clínico}

Desde una perspectiva anatómica, esta afasia es producto de una lesión en la región postcentral inferior izquierda (área 40 de Brodmann) que ocasiona, a su vez, una alteración en la base cinestésica de la articulación (Instituto Nacional de Rehabilitación, 2015, p. 3), por lo que los impulsos direccionales diferenciales, necesarios para los músculos del habla, se vuelven casi imposibles de realizar. Esto provoca que los movimientos de los músculos articulatorios pierdan su base aferente y, en consecuencia, se produzca una falta de precisión en los movimientos de la lengua y del aparato

\footnotetext{
2 El concepto de factor es fundamental dentro de la teoría de Luria, quien lo entendía como “'la función propia' de una u otra estructura cerebral [...] Cada zona cerebral que participa en la realización del sistema funcional, que se encuentra en la base de la función psicológica superior, es responsable de un factor determinado; su destrucción (o cambio patológico) conduce a la alteración del trabajo del sistema funcional correspondiente en general" (Xomskaya, 2002, p. 152). En el caso específico de la afasia, cada subtipo tiene un factor neuropsicológico alterado.
}

articulatorio, por lo que los articulemas se encuentran dañados (Luria, 1964).

Es común que estos pacientes presenten parafasias literales (sustituciones de fonemas), pero atendiendo a rasgos fonéticos particulares, esto es, tienden a sustituir un fonema por cercanía en punto o modo de articulación; por ejemplo, adelantar o atrasar el punto de articulación (producir /t/ por /k/), cambiar el modo de articulación (producir /b/ por $/ \mathrm{m} /$ ) o realizar un ensordecimiento (producir /p/ por /b/). No obstante, en casos severos, el paciente puede ser incapaz de realizar la combinación de movimientos necesarios para la pronunciación de un fonema (Quintanar et al., 2006). De acuerdo con Mejía, Solovieva y Quintanar (2002) también se encuentra comprometida la comprensión.

\section{Caracterización lingüística}

Esta afasia refleja un problema en la codificación ya que los pacientes sufren una dificultad para realizar la combinación de los distintos rasgos articulatorios que componen a los fonemas. Así pues, este conjunto de rasgos se vuelve tan complejo que los pacientes sólo emplean algunos de ellos y sustituyen otros de forma aleatoria. En este sentido, Jakobson (1971, p. 316) sostiene que los rasgos que se conservan son los que comunican la información fonológica, mientras que los sustitutos funcionan como meros rellenos. En breve, el déficit provoca la aparición de coalescencias, esto es, hay una "selección inadecuada de rasgos articulatorios que provoca que el paciente produzca un fonema por otro" (Ávila, 1977, p. 278). Este déficit se vuelve más profundo cuando el paciente realiza la tarea de manera voluntaria y consciente. 
Como consecuencia, se presenta una alteración en el lenguaje espontáneo, en la repetición y en la denominación (Quintanar et al., 2006; Galindo, Pelayo, Solovieva y Quintanar, 2014). En el caso de habla conectada, solamente está compuesta por palabras sueltas o frases porque el paciente está buscando constantemente "la posición necesaria del aparato fono-articulador para la pronunciación de los fonemas" (Instituto Nacional de Rehabilitación, 2015, p. 5), lo que entorpece y dificulta su producción. Justamente, estas características del lenguaje espontáneo podrían estar propiciando la alta aparición de formuladores.

Finalmente, en cuanto a las características discursivas, en específico de los marcadores del discurso, no hay trabajos previos que hayan abordado su estudio en hispanohablantes, principalmente por las serias dificultades que tienen estos pacientes para producir muestras amplias de habla espontánea. Sin embargo, sí existen estos estudios en otros tipos de afasia, principalmente en las fluidas; por ejemplo, Pietrosemoli, Vera, González Valera y Coutin Churchman (2005) analizan cuantitavimente el uso de estos elementos en tres grupos: (i) pacientes con daño cerebral derecho, (ii) pacientes con daño cerebral izquierdo y (iii) un grupo control. Los autores concluyen que existe una disminución en el uso total de los MD en los hablantes afásicos, aun cuando el marcador $y$ mantiene la misma frecuencia en todos los grupos. Así, según los autores, los MD contribuyen a la construcción de la cohesión y la coherencia, por lo que se conservan a pesar del déficit lingüístico presentado en otros niveles.

Por su parte, Sinacay (2013) estudió estas partículas en pacientes con afasia fluente, en particular: anómica, de conducción y de Wernicke. Encontró que funcionan como soportes de habla y facilitadores para el procesamiento del discurso y que muestran un comportamiento diferencial dependiendo del tipo de afasia de que se trate: en las afasias anómica y de conducción se encuentran mejor preservados, mientras que en la de Wernicke su uso es considerablemente limitado. Esto llevó a la conclusión de que cuando la afasia es leve, como en el caso de la anómica, la afección lingüística es menor y, por lo tanto, el uso de marcadores se mantiene mejor; en contraste, cuando la afasia es más severa, la afección lingüística es mayor y, en consecuencia, el uso de marcadores es menor. Finalmente, destaca que la propiedad polifuncional de los marcadores no se encuentra dañada en los pacientes con afasia porque dependen del valor pragmático-discursivo que les atribuye el hablante y que responde a los déficits comunicativos que padecen.

Guillén (2013) realizó un estudio contrastivo entre pacientes con afasia de Wernicke y hablantes sin patología. Se encontró que el uso de los marcadores discusivos no sólo se mantiene en los pacientes, sino que, además, son empleados en igual o mayor cantidad que en la producción del grupo control. Además, los marcadores que más se emplean son los que ayudan a subsanar los déficits comunicativos particulares de estos pacientes controladores de contacto, formuladores y reformuladores-, por lo que desempeñan una función compensatoria. Finalmente, la conclusión principal es que los MD se conservan porque son altamente funcionales y esto contribuye a que los 
hablantes afásicos mejoren sus habilidades comunicativas, tanto en la construcción del discurso como en el plano interaccional de la conversación.

Por último, el trabajo de Gallardo-Paúls y Marín Jordá (2005) es el único que se centra en pacientes con afasia no fluente, en particular: afasia de Broca (motora eferente). Los resultados de este trabajo confirman que los marcadores son una manifestación y una estrategia de cooperación conversacional porque le permiten al hablante afásico construir turnos productivos e iniciativos, aun cuando carezcan de contenido proposicional. En particular, hacen avanzar el tema del discurso, suplen deficiencias sintácticas y proposicionales, actuando como un mecanismo pragmático de compensación que intenta mantener atento al receptor, asegurar su escucha y su interpretación en términos argumentativos $y$, en menor medida, sirven para organizar el propio discurso del hablante con afasia.

Así pues, todos los trabajos destacan dos hechos: (i) que estas partículas discursivas se mantienen, en mayor o menor medida, dependiendo del tipo de afasia, y (ii) que tienden a ser empleadas para compensar los déficits lingüísticos particulares que impone esta patología. Con todo, aún no hay estudios centrados en la descripción del uso y de las funciones que desempeñan estas partículas en la afasia motora aferente. En consecuencia, una de las metas de este trabajo es contribuir a subsanar esta carencia y comenzar a describir las características discursivas de estos pacientes.

Tomando en cuenta lo expuesto hasta ahora, el objetivo de este trabajo es analizar cualitativamente el uso y las funciones de cuatro tipos de marcadores discursivos -conectores, reformuladores, formuladores y modalizadores- en la producción lingüística de un paciente con afasia motora aferente. Se busca mostrar que estos elementos lingüísticos se mantienen a pesar de la patología porque su uso responde, precisamente, a la compensación de los déficits comunicativos que padece el hablante. Se parte del supuesto, entonces, de que dado los problemas que se presentan en esta afasia, el uso productivo de estos marcadores estaría justificado.

\section{Presentación del caso}

El paciente J es un sujeto masculino de 33 años, con estudios universitarios, hablante nativo del español y de inglés como L2, lateralidad diestra y sin antecedentes psiquiátricos o neurológicos. J comenzó a tener problemas de expresión y dificultad para moverse por lo que fue trasladado al hospital donde permaneció siete días, después de haberle sido detectado un infarto cerebral. Como consecuencia, sufrió una pérdida de la marcha y de lenguaje, por lo que estuvo recibiendo tratamiento de rehabilitación física durante dos semanas. Los estudios tomográficos revelaron daño cortical y subcortical en la región central inferior izquierda, principalmente en zonas postcentrales. Finalmente, no presentaba dificultades para masticar o deglutir y dormía aproximadamente seis horas durante la noche $y$, en ocasiones, tomaba una siesta durante el día.

En cuanto a la evaluación neuropsicológica, se llevó a cabo en varias sesiones distribuidas entre el 8 y el 17 de agosto de 2016, en la Unidad Universitaria de Neuropsicología del Hospital Universitario de 
Puebla. Durante las sesiones se evaluaron los factores de integración cinestésico-táctil, organización cinética de los movimientos, retención visual y audio-verbal, oído fonemático, síntesis espaciales simultáneas y regulación y control de la actividad. Para tales fines, se aplicaron tres instrumentos: Diagnóstico clínico neuropsicológico de la afasia Puebla-Sevilla (Quintanar, Solovieva y León-Carrión, 2011), Evaluación neuropsicológica breve para adultos (Quintanar y Solovieva, 2009) y Evaluación neuropsicológica de la integración espacial (Quintanar y Solovieva 2012).

A partir de la evaluación neuropsicológica se encontró que el infarto provocó secuelas que afectaron el funcionamiento de zonas somatosensoriales -primarias y secundarias-. Este daño trajo consigo una alteración en el factor de integración cinestésica, lo que provocó una afasia motora aferente, que afectó todos los niveles de la actividad verbal expresiva e impresiva, tanto oral como escrita. También se encontró alterado, aunque en menor grado, el nivel de activación de trabajo cortical, lo que se ve reflejado en dificultades de retención en diversas modalidades (audioverbal, visual y táctil).

En cuanto a los déficits lingüísticos, el informe detalla que la compresión a nivel de palabra aislada se encuentra sin daño, mientras que en la denominación presenta dificultades en la emisión, aun cuando hace un adecuado reconocimiento de las palabras. También se observó que puede completar palabras si se le ayuda con la primera sílaba, pero en estos casos se presentan parafasias literales, por ejemplo "lagartisa" por "lagartija" o "portina" por "cortina". La producción oral se limita a nivel de palabras, principalmente sustantivos y frases estereotipadas ("no sé”, “¿cómo?, "sí", "no”, "ahorita", "a veces", "gracias", etcétera). La repetición verbal voluntaria no es posible. Finalmente, en cuanto a las tareas de identificación de sonido, de acuerdo con la grafía presentada, se observaron errores frecuentes relacionados con sustituciones de fonemas cercanos en punto de articulación principalmente.

\section{Método}

Como ya se mencionó, los datos fueron obtenidos en la Unidad Universitaria de Neuropsicología del Hospital Universitario de Puebla, después de la sesión de terapia neuropsicológica. Tanto J como su pareja fueron informados sobre la naturaleza de este trabajo, así como del manejo ético que se le daría a sus datos, por lo que consintieron, de manera expresa, la videograbación de la entrevista y el uso de ella para fines exclusivamente de investigación lingüística. Cabe destacar que se realizó una entrevista piloto para que el paciente se familiarizara con los investigadores y con el tipo de tarea que se iba a realizar.

El tipo de entrevista aplicada fue semiestructurada, donde los temas giraron en torno al padecimiento de J, su vida antes y después del infarto, así como las actividades que realiza durante el día. Debido a las características de la afasia que padece, era esperable que su producción fuera limitada por lo que el entrevistador dirigía la entrevista y trataba de estimular la participación del paciente, proponiendo temas y cooperando con la 
construcción de la conversación. Una vez concluida la entrevista, se procedió a su procesamiento informático. Para la transcripción de los datos se utilizó el programa ELAN (Sloetjes \& Wittenburg, 2008) y la convención de etiquetado del Grupo Val.Es.Co. (Briz y Grupo Val.Es.Co., 2002a, 2002b, 2003). Una vez realizado todo eso, se efectuó la búsqueda de los marcadores discursivos utilizados por J, y posteriormente ellos se agruparon de acuerdo con la función que desempeñan. Finalmente, para su inclusión ejemplar dentro del texto, se optó por una transcripción ortográfica.

\section{Resultados}

La entrevista cuenta con 258 turnos, en los cuales el paciente empleó un total de 1522 palabras, con una nómina de 70 ítems diferentes. La mayoría pertenece a las clases abiertas, en específico verbos, sustantivos, adjetivos y adverbios. En cuanto a los MD, el hablante los utilizó en 282 ocasiones para cumplir cuatro funciones distintas: (i) conexión, (ii) formulación, (iii) reformulación y (iv) modalización. Estos resultados se concentran en las Tablas 1 y 2.
Tabla 1

Densidad léxica de J

\begin{tabular}{llc}
\hline \multicolumn{2}{c}{ Densidad léxica de J. } \\
\hline Turnos & 258 \\
Total de palabras (token) & 1522 \\
Palabras distintas (type) & & 70 \\
\cline { 2 - 3 } Clases abiertas & Verbos & 19 \\
& Sustantivos & 12 \\
& Adjetivos & 4 \\
& Adverbios & 17 \\
Clases cerradas & Artículos & 2 \\
& Pronombres & 6 \\
& Preposiciones & 4 \\
& Conunciones & 6 \\
\hline
\end{tabular}

Tabla 2

Distribución y funciones de los marcadores discursivos empleados por J

\begin{tabular}{llc}
\hline Función & Marcador & Apariciones \\
\hline Conectores & $\begin{array}{l}\text { pero (92) } \\
\text { pues (12) } \\
\text { entonces (26) } \\
\text { después (2) } \\
\text { por eso (8) }\end{array}$ \\
& $\begin{array}{l}\text { porque (3 } \\
\text { bueno (3) } \\
\text { pues (2) } \\
\text { eh (42) } \\
\text { bueno (14) } \\
\text { Formuladores } \\
\text { este (18) } \\
\text { a ver (2) } \\
\text { bueno (38) } \\
\text { es que (20) }\end{array}$ \\
Modalizadores & 38 \\
Total & & \\
\hline
\end{tabular}

Así pues, a continuación se describe el uso de estos marcadores discursivos y las funciones que desempeñan en la producción lingüística del paciente. Para tal fin, se incluirán algunos ejemplos representativos de cada tipo de marcador.

\section{Conectores}

Esta función se empleó en 143 ocasiones por 
medio de 6 marcadores distintos: pero, pues, entonces, después, por eso y porque. Como se dijo antes, a través de esta función los hablantes señalan: (i) la orientación argumentativa, (ii) la organización discursiva, (iii) el inicio de un turno reactivo y (iv) las inferencias implícitas que debe realizar el oyente.

(9) E: ¿ya no siente dolor?

I: ajá, pero ahorita sí, ya no acá y ya no, entonces por eso ay pero... pero ya no

(10) E: muy bien, ¿y de niño qué hacía usted?

I: es que no sé, tú... pero tú tú, ah tú, ah ti... que por eso, por eso ya no [hablo] ${ }^{3}$

En (9), pero indica la contraargumentación: no siento dolor, pero ahorita sí [lo siento], así el marcador une dos argumentos con orientación contraria. Por su parte, en (10), por eso introduce la consecuencia de lo dicho en la unidad discursiva previa. En ambos casos, los marcadores están uniendo dos argumentos y guían el proceso inferencial que debe realizar el oyente.

(11) E: haga un intento de decirme, cuénteme usted, qué le pasó.

I: $\mathrm{mmm}$... eeh, orita este brazo... pero

E: primero se le durmió

I: ajá, después eeh, entonces por eso ay... $\mathrm{mmm}$... eeh, entonces no sé, es que no... es que me quedé

(12) E: cuénteme acerca de eso. Lo llevaron al hospital y ¿qué pasó?

\footnotetext{
${ }^{3}$ El hablante se lleva la mano a la boca haciendo un gesto traducible como "hablar". La información contenida en los corchetes corresponde a las inferencias que hace el entrevistador a partir de la gesticulación del hablante con afasia.
}

I: pues me, me y eeh no acá, por eso no sé... y tú ya no eso, ya no eso $\mathrm{mmm}$, entonces... pues no sé acá, no sé

En (11), el marcador trabaja en un nivel dialógico, dado que es el entrevistador quien inicia la secuencia discursiva con el marcador primero, mientras que el paciente sigue organizando el diálogo a través de los marcadores después y entonces. Lo mismo sucede en (12), en este caso en un plano monológico, el turno del paciente está organizado por diversos conectores: pues, por eso y entonces. En estos casos, los MD trabajan en la argumentación y en la organización del discurso del paciente. No obstante, la ausencia de léxico dificulta la comprensión del turno, aun cuando la estructura y la organización del discurso se mantenga preservada.

(13) E: usted ¿se acuerda en qué trabajaba? ¿Me puede decir?

I: mmm, sí, pero... [no lo puedo decir] ${ }^{4}$

(14) E: ok, ¿̇se acuerda de su trabajo, qué hacía? I: pues, vengo... que acá, cómo que... como, no sé es que...

E: no lo recuerda

I: no, no, no, me este es, sí, pero [no lo puedo decir]

En (13) y (14), los marcadores funcionan como generadores de las inferencias que debe realizar el oyente, es decir, el entrevistador debe completar las ideas comunicadas de manera implícita por el paciente. En (13), pero genera la inferencia "no me

\footnotetext{
${ }^{4}$ En los ejemplos 13 y 14, el hablante se lleva la mano a la boca, al tiempo que gira la cabeza de derecha a izquierda, haciendo ostensiva su incapacidad para hablar de manera fluida.
} 
va a entender", mientras que en (14) produce la inferencia "no lo puedo decir". Como característica general, cuando cumplen esta función, los marcadores se encuentran a final de turno y son seguidos por una pausa, incluso en varios casos se acompañan con movimientos corporales o gestos que ayudan a generar la inferencia. Este uso es bastante útil para el paciente, pues le permite comunicarse sin necesidad de emplear estructura lingüística, apelando al significado convencional de los marcadores.

\section{Reformuladores}

Esta función fue la que menos se empleó, tan sólo en 3 ocasiones y con el mismo marcador en todos los casos: bueno. Este hecho parece natural si recordamos que el paciente tiene problemas para la construcción de su intervención, esto es, no tiene que reformular lo que no puede construir.

(15) E: ¿y usted tiene hermanos? No le he preguntado

I: ah sí- bueno acá- bueno ya no, acá y tú

(16) E: muy bien. $Y$ dígame entonces, tiene planes, ¿qué va a hacer este fin de semana? ¿Hará algo?

I: mañana... ah no, mañana- bueno que esto pero no sé... a la, a la mente es que eso... eso a la, a la no

En ambos ejemplos el paciente edita y corrige su intervención al notar que ha dicho algo impreciso. En estos casos, el marcador no está antecedido por pausas.

\section{Formuladores}

Esta función apareció en 78 ocasiones, empleando 5 marcadores distintos: pues, eh, bueno, este y a ver. Como característica general, estos marcadores se encuentran entre pausas y vienen acompañados de alargamientos vocálicos o consonánticos, lo que le permite ganar más tiempo al paciente. Finalmente, en varias ocasiones aparecieron a inicio de turno, cumpliendo, así, una segunda función: marcar una intervención reactiva (respuesta).

(17) E: ¿cómo se encuentra? ¿bien? ¿qué le hicieron ahorita?

I: puees, $\mathrm{mmm}$... no sé, ees como quee eeh

(18) E13: aún le faltan palabras

I13: ajá sí, pero ya no mee mee costa, no... a ver, eeh no.

En (17), el paciente tiene problemas para formular su intervención por lo que emplea pues para ganar tiempo y pensar qué decir; además, también cumple con la función de introducir una intervención reactiva (respuesta). Estos problemas se ven reflejados también en los alargamientos vocálicos y en las pausas. En (18), el paciente emplea a ver para detenerse, pensar y buscar la posición correcta de los articulemas que quiere producir; de nuevo, la presencia de pausas y alargamientos vocálicos contribuyen a la construcción de su intervención.

\section{Modalizadores}

En términos generales, la actitud del hablante se encuentra incluida a lo largo de sus intervenciones, 
esto es, está consciente de los déficits que padece y, a pesar de lo comentarios positivos del entrevistador, la opinión del paciente con respecto a su situación es pesimista. Esto se ve reflejado en la gran cantidad de modalizadores de falso acuerdo que empleó: 25. Estos casos pueden ser parafraseados como: "sí, pero no".

(19) E: lo oí hace quince días y ya ahora habla más.

I: ah sí, ya, bueno pero... no sé, eeste...mmm, ¿ ¿cómo es? Ay, no sé.

(20) E: simplemente le cuesta trabajo hablar

I: sí, sí y todo, y sí, pero bueno [qué se le hace $]^{5}$

En (19) y (20), los marcadores bueno señalan el acuerdo parcial de paciente con respecto a lo que dice el entrevistador, esto se ve reforzado por la presencia de pero, que guía a una orientación contraria. El caso de (20) señala, incluso, la resignación del paciente ante su condición.

En otros casos, la modalización refleja un acuerdo genuino entre el paciente y el entrevistador, con lo que se marca la disposición del paciente a participar en la conversación:

[Hablando sobre equipos de futbol]

(21) E: al Barcelona, ¿no?

I: ah bueno, sí

Finalmente, el paciente utiliza el marcador es que para justificar su opinión, más que para dar una razón o un argumento.

${ }^{5}$ El hablante se encoje de hombros y el semblante se torna apesadumbrado.
(22) E: y bien, entonces ya lo entienden más I: ajá, sí pero orita es que me quedo y tú eso, me quedo... ajá

Así pues, el uso de marcadores discursivos es constante y productivo en el habla de nuestro paciente. Como se ha venido abogando, esto es así porque le ayudan a cubrir: (i) las carencias en la formulación de la intervención, (ii) las deficiencias sintácticas y (iii) la ausencia de léxico. A continuación se ahondará en esto.

\section{Discusión y conclusiones}

En este trabajo se analizó el uso de cuatro tipos de marcadores discursivos en la producción lingüística de un paciente con afasia motora aferente. En primer lugar, se debe decir que la producción del paciente es limitada en cuanto a longitud y en cuanto a contenido proposicional explícito, pero el uso de los conectores le ayuda a producir inferencias implícitas que facilitan su participación a lo largo del intercambio comunicativo, ya que le ahorran el uso de estructura lingüística. En particular, se observó que el paciente recurre a una fórmula común: emplea consistentemente el marcador pero para hacerle ostensivo al interlocutor que, a pesar de entender y querer hablar, no puede articular de manera fluida ni responder adecuadamente a las preguntas.

Así pues, el uso de marcadores discursivos se mantiene y resulta bastante productivo para el paciente, pues le ayuda a lidiar con los déficits que le impone la afasia. En este sentido, los resultados 
coinciden con las investigaciones previas al considerar que los marcadores cumplen con una función de compensación ante la comunicación deficitaria que se presenta en la afasia. Esto es, el tipo de marcador empleado responde a las características del discurso del paciente, por lo que si hay un uso generalizado de formuladores es porque en este tipo de afasia existen particularmente problemas en la construcción del turno comunicativo, dado los déficits motores. Lo que hay que destacar aquí es que el paciente lo sabe y le señala ostensivamente al interlocutor que está tratando de cubrir estas dificultades aun cuando no pueda hacerlo del todo.

Además, considerando los estudios previos, tal y como lo señala Sinacay (2013), existe un uso diferenciado de los MD que depende del tipo de afasia del que se trate. Así, por ejemplo, parece lógico que los déficits que tenga que compensar un paciente con afasia fluida no serán los mismos que tenga que subsanar uno con afasia no fluida, y esto se ve reflejado en el tipo de MD empleado.

Por otra parte, una explicación común para la conservación de este tipo de elementos lingüísticos es que se trata de estereotipos o automatismos y que el paciente afásico los produce porque el programa motor para producirlos se encuentra consolidado, esto es, los articulemas están bien aprendidos y no tiene que lidiar con la búsqueda de los movimientos particulares para su producción. No obstante, esto es sólo una explicación parcial; más bien, estos marcadores se producen porque son altamente funcionales en el discurso afásico y, como factor secundario, el programa motor podría tener una ruta bastante bien aprendida. Así pues, su aparición abundante se podría justificar, en primer lugar, por su alta funcionalidad y, en segundo lugar, esto provocaría que el programa motor se automatice a partir del uso constante.

También es destacable que la frecuencia de los marcadores sea tan basta, esto coincide con la apreciación de Gallardo-Paúls y Marín Jordá (2005) sobre que suplen deficiencias proposicionales actuando como mecanismo pragmático de compensación, esto es, aunque el contenido proposicional no exista, la estructura discursiva del turno del paciente está presente y señala, al menos, (i) el proceso inferencial que debe seguir el interlocutor y (ii) la organización discursiva del turno del paciente.

Finalmente, de acuerdo con los resultados, se puede adelantar que los marcadores funcionan para que el paciente: (i) señale el inicio de su turno, como introductor de intervención reactiva, (ii) justifique su opinión, (iii) introduzca inferencias, (iv) corrija sus movimientos, (v) mantenga el turno, (vi) subsane los problemas durante la construcción del discurso, (vii) organice estructuralmente su intervención e (viii) indique un falso acuerdo y un acuerdo genuino. Todo esto manifiesta que, en el plano interaccional, el paciente marca la toma de turnos y señala un acuerdo; en el plano monológico, organiza y edita la construcción de su intervención, $y$, en el plano enunciativo, justifica sus opiniones.

Con todo, es importante mencionar que este es un primer acercamiento al estudio del uso de los marcadores discursivos en la afasia motora aferente. Aun más, dado que es un estudio de caso, no se pueden establecer generalizaciones significativas; más bien, estos resultados se postulan como 
hipótesis interpretativas que deberán ser comprobadas a partir de estudios con muestras más amplias. Finalmente, se secundan las opiniones de Pietrosemoli (1994) y Gallardo-Paúls (2009), el nivel discursivo en la afasia muestra una desintegración menos severa que otros niveles lingüísticos, por lo que se deberían plantear descripciones más adecuadas de la capacidad lingüística general en la afasia y no sólo limitarse a los aspectos del lenguaje más tradicionales. Estos nuevos aspectos incluirían, entre otras cosas, la descripción de las habilidades conversacionales, comunicativas e interactivas en la afasia, aspectos que han demostrado estar mejor preservados y servir como estrategias compensatorias en el habla deficitaria que se presenta en la afasia.

\section{Referencias}

Anscombre, J.C. y Ducrot, O. (1994). La argumentación en la lengua. Madrid, Gredos.

Ardila, A. (2005). Las afasias. Guadalajara: Universidad de Guadalajara.

Ávila, R. (1977). Afasias: selección, combinación, signos y fonemas. Nueva Revista de Filología Hispánica, 26(2), 276285.

Basso, A. (2003). Aphasia and Its Therapy. Oxford: Oxford University Press.

Briz, A. (1993). Los conectores pragmáticos en español coloquial (II): su papel metadiscursivo. Español Actual, 59, 39-56.

Briz, A. (2008). "Presentación”, en Diccionario de Partículas Discursivas del Español (DPDE), disponible en: «www.dpde.es» Servei de Publicacions de la Universitat de València.

Briz, A. y Grupo Val.Es.Co (2002a). Corpus de conversaciones coloquiales. Madrid: Arco-libros.

Briz, A. y Grupo Val.Es.Co (2002b). La transcripción de la lengua hablada: el sistema del Grupo Val.Es.Co. Español Actual, (77-78), 57-86.

Briz, A. y Grupo Val.Es.Co (2003). Un sistema de unidades para el estudio del lenguaje coloquial, Oralia, 6, 7-61.

Briz, A. y Pons, S. (2010). Unidades, marcadores y posición. En O. Loureda y E. Acín (Coords.). Los estudios sobre marcadores del discurso, hoy. (pp. 523-557). Madrid: Arco.

Briz, A., Pons, S. y Portolés, P. (2008). Diccionario de partículas discursivas del español (DPDE) [en línea]. Disponible en: http://www.dpde.es.

Galindo, G. M., Pelayo, H., Solovieva, Y. y Quintanar L. (2014). Rehabilitación neuropsicológica en un caso de afasia motora aferente. Pensamiento Psicológico, 12(2), 97-112.

Gallardo-Paúls, B. (2009). Valoración del componente pragmático a partir de datos orales. Revista de Neurología, 48(2), 57-61.

Gallardo-Paúls, B. y Marín Jordá, J. (2005). Marcadores discursivos procedentes de verbos perceptivos en el discurso afásico. Revista de Investigación Lingüística, 8, 53-94.

Geschwind, N. (1976). Language and the brain. En R. Thompson (Ed.), Progress in psychobiology (pp. 341-348). New York: Scientific American.

Goodglass, H. y Geschwind, N. (1976). Language disorders (aphasia). En E. Carterete \& M. Friedman (Eds.), Handbook of perception, Vol. VII: Language and speech (pp. 389428). New York: Academic Press.

Guillén, J. (2013). El uso de marcadores discursivos y las funciones que desempeñan en pacientes con afasia de Wernicke. Un estudio constrastivo (Tesis doctoral, EI Colegio de México, México). Disponible en: https://www.academia.edu/20096883/El_uso_de_marcad ores_discursivos_y_las_funciones_que_desempeñan_dent ro_de_las_producciones lingü\%C3\%ADsticas_de_paciente s_con_afasia_de_Wernicke._Un_estudio_contrastivo

Instituto Nacional de Rehabilitación (INR). (2015). Manual de guía clínica de la afasia motora aferente. México: 
Secretaría de Salud.

Jakobson, R. (1971). Selected Writings II. Word and Language. Mouton The Hague: París.

Luria, A.R. (1964). Factors and Forms of Aphasia. En A. De Reuck y M. O'Connor (Eds.), Ciba Foundation Symposium on Disorders of Language (pp. 143-161). Londres: J. \& A. Churchill Ltd.

Luria, A.R. (1977). Las funciones corticales superiores del hombre. La Habana: Orbe.

Mejía, B., Solovieva, Y. y Quintanar, L. (2002). Alteraciones de la comprensión del lenguaje oral en la afasia motora aferente y en la afasia mixta. Cuadernos Hispanoamericanos de psicología, 2(1), 53-66.

Portolés, J. (1998). Marcadores del discurso. Barcelona: Ariel.

Pons, S. (1998). Oye y mira o los límites de la conexión, en Ma Antonia Martín Zorraquino y Estrella Montolío Durán (Eds.), Marcadores discursivos: teoría y práctica (pp. 213228). Madrid: Arco.

Pons, S. (2001). Connectives/Discourse Makers. An Overview. En Ferrer Mora, Hang y Pons Bordería, Salvador (Eds.) La pragmática de los conectores y las partículas modales (pp. 219-243). Valencia, Quaderns de Filologia. Estudis Literaris, VI.

Pons, S. (2003). From agrement to stressing and hedging: Spanish bueno and claro. En G. Held (Ed.), Partikeln und Höflichkeit (219-236). Bern: Peter Lang.

Pietrosemoli, L. (1994). Coherencia y cohesión en el discurso afásico. Lengua y Habla 1, 78-88.

Pietrosemoli, L., Vera, M., González Valera, S. y Coutin Churchman, P. (2005). Marcadores discursivos en hablantes sanos y afásicos: El caso especial de y. Boletín de Lingüística, 17(24), 25-30.

Quintanar, L., Lázaro García, E. y Solovieva, Y. (2006). Diagnóstico neuropsicológico de la afasia motora aferente. Revista Española de Neuropsicología, 8(1-2), 43-61.

Quintanar, L. y Solovieva, Y. (2009). Evaluación neuropsicológica breve para adultos. Puebla: Benemérita Universidad Autónoma de Puebla.
Quintanar, L., Solovieva, Y. y León-Carrión, J. (2011). Evaluación neuropsicológica clínica de las afasias PueblaSevilla. Puebla: Benemérita Universidad Autónoma de Puebla.

Quintanar, L. y Solovieva, Y. (2012). Evaluación neuropsicológica de la integración espacial [Manuscrito no publicado]. Puebla: Universidad Autónoma de Puebla.

Schiffrin, D. (1987). Discourse Markers. Cambridge: Cambridge University Press.

Sinacay, E. (2013). Descripción de marcadores del discurso de hablantes con afasia fluente: anómica, de conducción y de Wernicke (Tesis de maestría, Pontificia Universidad Católica del Perú, Perú). Recuperado desde: http://tesis.pucp.edu.pe/repositorio/handle/123456789/5 $\underline{034}$

Sloetjes, H., \& Wittenburg, P. (2008). Annotation by category - ELAN and ISO DCR. En: Proceedings of the 6th International Conference on Language Resources and Evaluation.

Valdois, S., Joanette, Y., Nespoulous, J. \& Poncet, M. (1988). Afferent Motor Aphasia and Conduction Aphasia. En H. Whitaker (Ed.), Phonological Processes and Brain Mechanisms (pp. 59-92). Nueva York: Springer-Verlag.

Xomskaya, E. (2002). El problema de los factores en la neuropsicología. Revista Española de Neuropsicología, 4(23), 151-167. 